\title{
Las mujeres agentes en la producción científica: el caso de la educación en Chihuahua
}

\author{
Romelia Hinojosa Luján \\ Secretaría de Educación, Cultura y Deporte del Estado de Chihuahua, \\ Red de Investigadores Educativos Chihuahua, A.C. \\ rome_hinojosa@yahoo.com.mx
}

\begin{abstract}
Resumen
Este trabajo plantea los hallazgos realizados a través del Diagnóstico Estatal de la Investigación Educativa que se emprendió a través de la Secretaría de Educación Cultura y Deporte (SECyD) y la Red de Investigadores Educativos Chihuahua, AC (REDIECH). La información se reunió a partir de cuatro importantes fuentes: un cuestionario electrónico con invitación amplia para responderlo, los Estados de Conocimiento de la Investigación Educativa que la misma SECyD, las tesis de las instituciones que ofertan posgrados pedagógicos de la entidad y las memoria de los Congresos Nacionales de Investigación Educativa que realiza cada dos años el Consejo Mexicano de Investigación Educativa. Si bien el diagnóstico fue amplio, este escrito recupera únicamente la información sobre los agentes productores de la misma y el análisis se centra en la categoría de género. Los hallazgos muestran que la desigualdad cultural que sufren las mujeres afecta su participación en el campo y la producción científica. Es uno de los capitales importantes a considerar para caracterizar el trabajo de los agentes de la investigación educativa.
\end{abstract}

Palabras clave

Campo de la educación, investigadoras, género.

\section{Introducción}

La sociedad es una construcción social (Berger and Luckman, 2003): el lenguaje, las costumbres, la vestimenta, las relaciones... es decir, todo por completo, está mediado por la producción que hombres y mujeres han realizado y realizan de ella. Los determinantes socioculturales originan comprensiones cognitivas diferenciadas entre hombres $\mathrm{y}$ mujeres.

El orden sociocultural configurado sobre la base de la sexualidad -definida históricamente- se expresa a través del género categoría que permite comprender las asignaciones y expectativas socioculturales hacia las personas en dependencia de sus diferencias sexuales. Implica las actividades y creaciones de los sujetos, el hacer en el mundo, la intelectualidad y la afectividad, el lenguaje, concepciones, valores, el imaginario, las fantasías, los deseos, la identidad, autopercepción corporal y subjetiva, el sentido de sí mismo, los bienes materiales y simbólicos, los recursos de vitales, el poder del sujeto, la capacidad para vivir, posición social, jerarquías estatus, relación con otros, oportunidades, el sentido de la vida y los límites propios (Fernández, 2005).

Cada mujer y cada hombre son la cristalización de lo que la sociedad en conjunto hace y recrea en ellos, son sujetos del proceso sociocultural a los límites impuestos por el mundo social. Algunos de estos límites que la sociedad impone, son precisamente los principios del género. Claro que es necesario asumir también, que cada uno de ellos y de 
ellas participa activamente en su delimitación como personas y en su identidad de género. Son delimitados, pero a la vez tienen márgenes de libertad en los cuales ellos y ellas toman decisiones. Esto origina tensiones y contradicciones.

Estas relaciones de género están implícitas en todas las actividades sociales emprendidas por los seres humanos. Es el caso de la ciencia y su producción. El conocimiento científico ha sido entonces construido por las relaciones de poder, por lo económico, lo social, histórico, lo político y desde la visión masculina. La racionalidad, la objetividad, la ausencia de subjetividad, de intimidad, la exactitud, lo medible y cuantitativo es lo que caracteriza entonces a la ciencia. Se exenta de emociones, de subjetividades, de intuición, sentimientos, porque eso es "lo otro", lo "no científico", "lo femenino". Las mujeres son lo otro en la ciencia, puesto que son intuitivas, se rigen por los sentimientos $y$, a partir de la caracterización del modelo masculino, quedan excluidas del saber y de la producción científica.

El sesgo androcéntrico se observa a nivel estructural en el escaso acceso de la mujer a la producción científica y en las barreras que encuentran las mujeres para ocupar puestos decisivos en las directrices de la ciencia y la tecnología, así como en la elaboración del discurso científico (Colás, 2001).

"La perspectiva de género opta por una concepción epistemológica que se aproxima a la realidad desde las miradas de los géneros y sus relaciones de poder" (Gamba, 2008). Es una tarea importantísima revisar los fenómenos de producción del conocimiento en lo general y el conocimiento educativo en lo particular con una perspectiva de género.

El acceso a las universidades ha surgido como un espacio de exclusión, si recordamos que es hasta los albores del siglo $\mathrm{XX}$ cuando las mujeres se empiezan a aceptar en estas instituciones (Blazquez and Flores, 2005). Sin embargo, la entrada de las mujeres a la educación superior no ha asegurado la igualdad en el campo productivo (Zubieta et al., 2005), ni la producción y generación del conocimiento (Blazquez and Flores, 2005, DelVal, 2005, Ríos, 2005).

De manera contraria al fenómeno de la representación femenina en la vida pública, desde los finales del siglo XIX y principios del XX un número muy importante de mujeres ha incursionado en la docencia. Este trabajo es uno de los espacios socialmente aceptados para que las mujeres incursionen en el ámbito productivo. Si se juzga de manera inocente el fenómeno se asumiría como natural el fenómeno de la feminización de la enseñanza. Sin embargo tiene también aspectos de género que a la simple luz no se perciben: es un trabajo ligado de manera "natural" con la mujer porque es una extensión del cuidado materno, sus horarios de trabajo permiten ejercer "adecuadamente" la maternidad o el cuidado de un hogar propio, al ser un nicho donde las mujeres se han desarrollado profesionalmente se encuentran relacionados con profesiones en el que el sueldo es bajo precisamente porque son profesiones "femeninas". Es pues el campo de la educación dominado numéricamente por las mujeres. Analicemos si este fenómeno está presente en la producción científica a través del seo y las cargas culturales a partir del mismo que privan en el campo de la investigación educativa.

\section{Desarrollo}

Los agentes de la investigación educativa son todas aquellas personas que transitan en el campo de la producción del conocimiento científica que tiene por objeto de estudio la educación. El criterio para delimitar la categoría de agente de la investigación educativa fue incluyente, se tomó como única condición tener algún producto de investigación educativa en la entidad. 
La producción generada permitió ubicar a los agentes en cuatro categorías: potenciales, en desarrollo, en consolidación y consolidados.

Los agentes potenciales de la investigación se definen como aquellos que registraron uno o dos productos (que en su mayoría son una tesis de maestría y una ponencia). Estos agentes se consideran potenciales pues aunque han vivido una experiencia de investigación aún no han incursionado en el campo.

Los agentes en desarrollo son aquellos que ya han incursionado de manera incipiente en el campo de la investigación, así que registran entre tres y seis productos, que en una generalidad se traducen en tesis de posgrado, ponencias y algunos artículos de revista.

Como agentes en consolidación se denomina a aquellos que registran entre siete y nueve productos de investigación. Se les considera en consolidación por su permanencia y debate en el campo, pues algunos de ellos tienen pocos años de incursionar en el ámbito de la investigación educativa cuentan con una diversidad de productos a través de los cuales establecen el debate con sus pares.

Finalmente, se clasifica como agentes consolidados a aquellos que registran diez o más productos de investigación. Generalmente son investigadores que tienen mayor antigüedad en el campo y han difundido su producción por una gran diversidad de medios. Algunos de ellos cuentan con reconocimiento social e institucional por su quehacer, pues perteneces al Sistema Nacional de Investigadores (SNI) y al Consejo Mexicano de Investigación Educativa (COMIE).

$\mathrm{Al}$ analizar a los agentes del campo de la investigación educativa en Chihuahua de acuerdo al género, se encontró que la gran mayoría de los agentes que están produciendo investigación son mujeres, pues de un total de 646 , las mujeres representan un $64 \%$ y el $36 \%$ son varones. La distribución según el nivel de producción se muestra a continuación.

Tabla 1. Agentes de la investigación por categoría y género.

\begin{tabular}{|l|l|l|l|}
\hline INVESTIGADORES & MUJERES & HOMBRES & TOTAL \\
\hline Potenciales & 391 & 216 & 608 \\
\hline En desarrollo & 13 & 11 & 24 \\
\hline En consolidación & 5 & 4 & 9 \\
\hline Consolidados & 2 & 3 & 5 \\
\hline TOTAL & 413 & 233 & 646 \\
\hline
\end{tabular}

En la generalidad de los agentes de la investigación educativa se aprecia el dominio femenino en la producción en el campo. Sin embargo, un análisis fino de la presencia femenina en las categorías más consolidadas de la investigación, evidencia cómo al final de éstas el predominio es masculino. En los agentes potenciales, en desarrollo y en consolidación, la distribución de la población es similar a la generalidad del campo, pero en la categoría consolidados se encuentra que un $40 \%$ son mujeres y un $60 \%$ de hombres, lo cual evidencia que los agentes que tienen mayor producción y permanencia en el campo 
de la investigación, así como un acceso mayor y diverso a los medios para difundir el conocimiento, son hombres.
Al realizar el análisis de las formas de difusión por género se encontró la siguiente distribución.

Tabla 2. Los agentes de la investigación por género y la forma de difusión de sus productos.

\begin{tabular}{|l|l|l|l|}
\hline $\begin{array}{l}\text { TIPO DE } \\
\text { PRODUCTO }\end{array}$ & HUJERES & TOTAL & \\
\hline Ponencias & 20 & 18 & 38 \\
\hline Capítulos de libro & 3 & 3 & 6 \\
\hline Cuadernos de trabajo & 1 & 0 & 1 \\
\hline Artículos de & 8 & 6 & 14 \\
\hline $\begin{array}{l}\text { Informes } \\
\text { investigación }\end{array}$ & 14 & 13 & 27 \\
\hline Libros & 4 & 6 & 10 \\
\hline
\end{tabular}

El análisis de las formas de difusión por género muestra que existe una homogeneidad en cada una de ellas con la generalidad del campo. La única diferencia encontrada, aunque es mínima, es la desventaja de las mujeres con respecto a los varones en la publicación de libros. Al igual que en la generalidad del campo, para entender este suceso habría que hacer un análisis exhaustivo de las condiciones del campo de la investigación educativa. Si acompañamos al análisis con la idea de que existen más mujeres en el campo de la producción científica sobre la educación, podemos dimensionar de mejor manera la desigualdad que las cifras en paridad, representan.

Al analizar las formas de producción por género se encontró lo siguiente:

Tabla 3. Los agentes de la investigación por género y las formas de producción.

\begin{tabular}{|l|l|l|l|}
\hline $\begin{array}{l}\text { FORMAS DE } \\
\text { PRODUCCIÓN }\end{array}$ & MUJERES & HOMBRES & TOTAL \\
\hline Individual & 4 & 4 & 8 \\
\hline Grupal & 2 & 1 & 3 \\
\hline Individual / Grupal & 14 & 13 & 27 \\
\hline TOTAL & 20 & 18 & 38 \\
\hline
\end{tabular}

Como se puede observar en la tabla hay homogeneidad en las formas de producción sin importar el género. Es decir tanto los investigadores como investigadoras utilizan las mismas estrategias de producción. Tanto en los que se agrupan como en los que 
trabajan en solitario o que combinan ambas modalidades.

Para el estudio de la permanencia de los agentes en el campo según su género, se tomaron como referencia los intervalos anteriores y la distribución de la población quedó de la siguiente manera

Tabla 4. Permanencia de los agentes en el campo según su género.

\begin{tabular}{|l|l|l|l|}
\hline $\begin{array}{l}\text { AÑOS PRODUCIENDO EN } \\
\text { EL CAMPO }\end{array}$ & HOMBRES & MUJERES & TOTAL \\
\hline De 1 a 5 & 3 & 3 & 6 \\
\hline De 6 a 10 & 6 & 7 & 13 \\
\hline De 11 a 15 & 3 & 5 & 8 \\
\hline De 16 o más & 5 & 6 & 11 \\
\hline TOTALES & 17 & 21 & 38 \\
\hline
\end{tabular}

La distribución por género evidencia que al inicio del periodo analizado, las cifras entre hombres y mujeres que incursionan en el campo son similares. Al permanecer entre $6 \mathrm{y}$ 10 años en el campo, la cifra entre hombres y mujeres se inclina hacia las mujeres, que representan un $57 \%$ y los varones se quedan en un $43 \%$.

Los datos también muestran que en el periodo de 16 años o más, las mujeres tienen una mayor permanencia, representan un $63 \%$. Sin embargo, cuando se analiza este aspecto entre quienes tienen mayor permanencia en el campo, el porcentaje de las mujeres disminuyen a un $55 \%$, por lo que el género masculino tiende a recuperar representatividad con un 45\%. Aun así la mujer sigue posicionada en la permanencia del campo.

Con la finalidad de conocer con detalle el cuerpo de agentes que pertenecen a las distintas instituciones, se realiza un análisis del número de agentes que pertenece a cada institución según su género. La distribución de la población se muestra en la siguiente tabla: 
Tabla 5. Agentes de la investigación por género y la institución de adscripción.

\begin{tabular}{|l|l|l|l|}
\hline INSTITUCIONES & MUJERES & HOMBRES & TOTAL \\
\hline CCHEP & 3 & 3 & 6 \\
\hline CD & 5 & 4 & 9 \\
\hline IBYCENECH & 1 & 0 & 1 \\
\hline ITESM & 0 & 2 & 2 \\
\hline UACH & 4 & 5 & 9 \\
\hline UACJ & 1 & 1 & 2 \\
\hline UPN & 5 & 0 & 5 \\
\hline SECYD & 0 & 2 & 2 \\
\hline NORMAL SAUCILLO & 0 & 1 & 1 \\
\hline INDEPENDIENTE & 1 & 0 & 1 \\
\hline TOTAL & 20 & 18 & 38 \\
\hline
\end{tabular}

La distribución de los agentes de la investigación educativa según su género se presenta de manera homogénea en las diferentes instituciones, aproximadamente están distribuidos en $50 \%$ de cada sexo. La única distribución atípica se presenta en la UPN, que cuenta con agentes sólo del sexo femenino. Esto permite inferir que el género no es un aspecto significativo para clasificar el quehacer de los agentes dentro de las instituciones. Nuevamente hay que traer a la memoria el fenómeno de feminización de la docencia para poder ver más allá de las cifras "iguales" y entender que lo justo, a partir de la feminización del campo, es que hubiese en todas las instituciones más mujeres que hombres.

Un aspecto importante que se puede rescatar dentro del análisis del capital institucional de los agentes estudiados, es la pertenencia a organismos que otorgan un reconocimiento social en las tareas de investigación, tales como el Sistema Nacional de Investigadores (SNI) y el Consejo Mexicano de la Investigación Educativa (COMIE). No obstante, este rubro no se desglosa a profundidad en este apartado por la escasez de agentes que pertenecen a estas instancias académicas, pues sólo dos de los 38 agentes pertenecen a ambos organismos, y otros dos son miembros sólo del COMIE, de tal manera que el reconocimiento social de los agentes que realizan investigación educativa en el estado de Chihuahua es muy precario en el contexto nacional. Ninguno de ellos es mujer, todos son hombres. El reconocimiento externo de los agentes en el campo, es enteramente dominado por los hombres. En este aspecto evidencia las dificultades que las mujeres tienen que sortear para recibir este tipo de estímulos o reconocimientos.

En el contexto nacional, la representatividad de los agentes investigadores educativos en los congresos nacionales que organiza el COMIE (IV, V, VI, 
VII) está cargada hacia las investigadoras, en un 60\% (Osorio, s/d).

Tabla 6 Congresos Nacionales de Investigación Educativa y participación femenina.

\begin{tabular}{|l|l|l|l|}
\hline $\begin{array}{l}\text { Congreso Nacional de } \\
\text { Envestigación }\end{array}$ & $\begin{array}{l}\text { Fúmero de Ponencias } \\
\text { presentadas por } \\
\text { mujeres }\end{array}$ & $\begin{array}{l}\text { Porcentaje de la } \\
\text { participación femenina }\end{array}$ \\
\hline IV & $\begin{array}{l}29 \text { al } 31 \text { de octubre } \\
1997\end{array}$ & 113 & $45 \%$ \\
\hline V & $\begin{array}{l}31 \text { de octubre al } 1^{\circ} \text { de } \\
\text { noviembre de } 1999\end{array}$ & 340 & $53 \%$ \\
\hline VI & $\begin{array}{l}6 \text { a } 10 \text { de noviembre } \\
\text { de } 2001\end{array}$ & 227 & $60 \%$ \\
\hline VII & $\begin{array}{l}18 \text { al } 22 \text { de noviembre } \\
\text { de } 2003\end{array}$ & 263 & $63 \%$ \\
\hline
\end{tabular}

Fuente Osorio (s/d).

Se aprecia que la participación femenina va en aumento, alcanza la paridad y desbanca la participación masculina. Sin embargo esta cifra no es proporcional a lo feminizado que está el campo educativo en lo general, aunque sí es proporcional a la membresía del COMIE. Vale destacar que en los CNIE no participan sólo los miembros de la misma, sino que entran en juego otros agentes que producen investigación educativa de todo el país.

En el caso de Chihuahua, se tiene el registro pormenorizado de la presentación de ponencias realizadas por autoras y autores en los Congresos Nacionales de Investigación Educativa (CNIE) VII, VIII, IX, X y XI.

Gráfica 1.

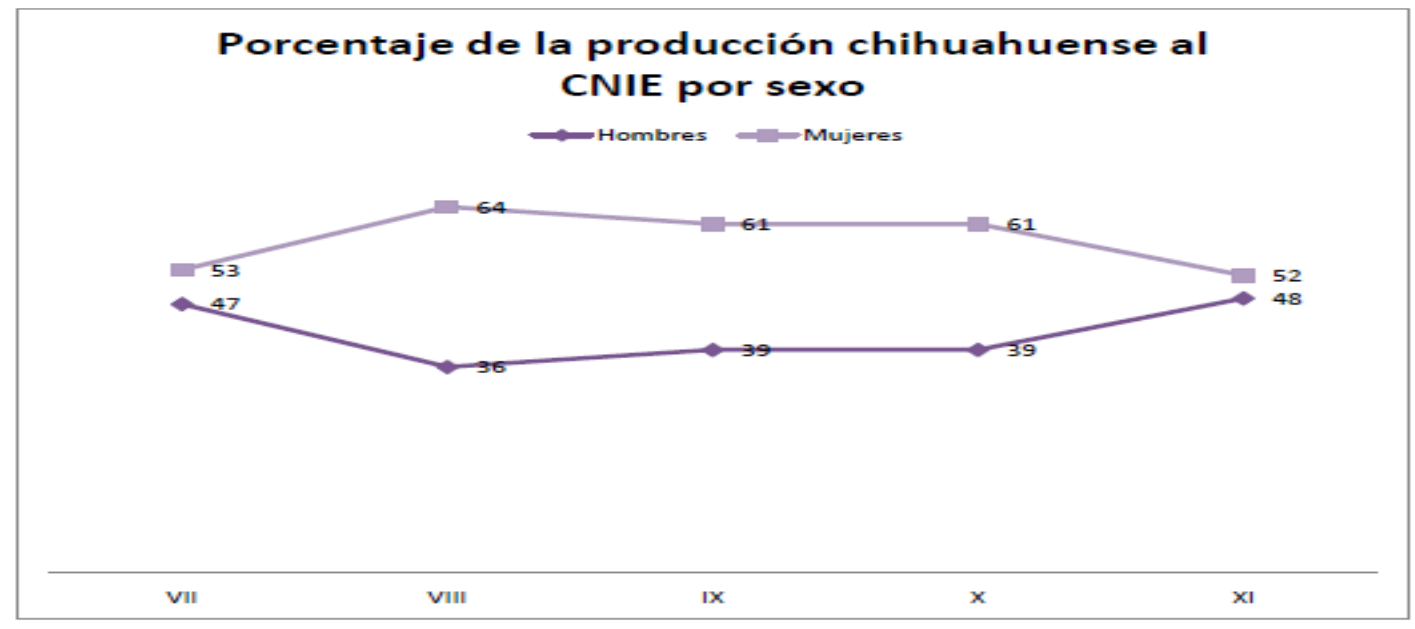

Hinojosa Luján, R. 
Fuente: Memorias de los Congresos Nacionales de Investigación Educativa.

La producción chihuahuense del VII CNIE es casi igualitaria entre hombres y mujeres. Sube la productividad de las mujeres durante tres congresos y en el último vuelve a representar otra vez casi el $50 \%$ para cada uno de los sexos. Nuevamente el fenómeno, visto de manera simple se podría decir que en Chihuahua las cifras reflejan la paridad con la que la cultura de género ha influido en hombres y mujeres, sólo que se olvida que las mujeres representan en el mundo educativo casi el $75 \%$.

Sobre la producción en las áreas designadas por COMIE para categorizar la temática de la investigación educativa, un análisis de las ponencias presentadas por Chihuahua en los CNIE refleja la feminización de algunas áreas como Currículo, Aprendizaje y Desarrollo Humano, Educación y Valores entre otros. Esto se observa en la Gráfica 2.

Gráfica 2.

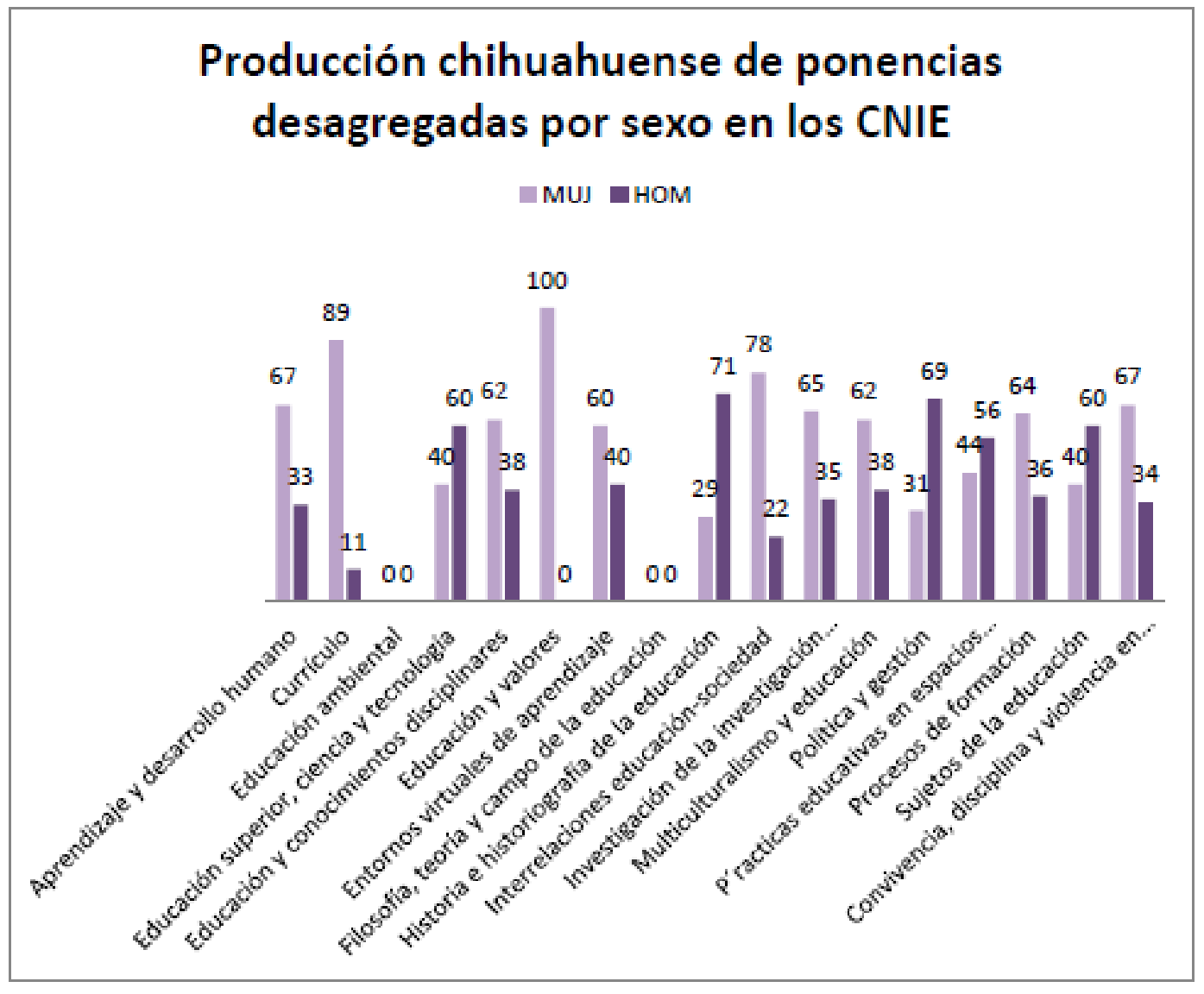

Fuente: Memorias de los congresos.

La gran ausente en la producción del campo es Filosofía, Teoría y Campo de la Educación en la que no hay producción estatal. Llama la atención el área de Política y Gestión donde casi el 70\% de la producción es realizada por hombres; Sujetos de la Educación y Educación Superior Ciencia y Tecnología en las que en las dos terceras partes es dominio varonil. Esto habla de la separación que se realiza de las parcelas de la realidad por género.

\section{Conclusiones}

El campo de la investigación educativa está cuantitativamente dominado por las mujeres. 
No es así, cuando se entra en cruce la variable del reconocimiento y prestigio social: los hombres dominan éste. Es decir el campo de la investigación educativa en la entidad está conformado mayoritariamente por mujeres, pero no está dirigido por ellas, ni representado al momento de la producción y de la difusión. Por otro lado, se percibe también en las áreas que establece el COMIE que hay parcelas "propicias" para las mujeres y otras que parecen "exclusivas" de los hombres.

Existe una imperiosa necesidad social de elaborar estrategias que modifiquen las condiciones sociales e institucionales para hacer posible que las mujeres participen activa y suficientemente en la producción de la ciencia. Se está desperdiciando el talento de millones de ellas y la mirada innovadora que la diferencia aporta.

\section{Referencias}

BERGER, P. \& LUCKMAN, T. 2003. La Construcción Social de la Realidad, Argentina, Amorrortu.

BLAZQUEZ, N. \& FLORES, J. 2005. Género y ciencia en América Latina. El caso de México. In: BLAZQUEZ, N. \& FLORES, J. (eds.) Ciencia, Tecnología y Género en Iberoamérica. México: UNAM.

COLÁS, P. 2001. Educación e investigación en la sociedad del conocimiento: enfoques emergentes. Revista Mexicana de Investigación Educativa, 19, 291-313.

DELVAL, C. 2005. Luces y sombras en torno a la participación de las mujeres en la investigación científica. In: BLAZQUEZ, N. \& FLORES, J. (eds.) Ciencia, Tecnología y Género en Iberoamérica. México: UNAM.

FERNÁNDEZ, L. 2005. Género y mujeres académicas: ¿Hasta dónde la equidad? In: BLAZQUEZ, N. \& FLORES, J. (eds.) Ciencia, tecnología y género en Iberoamérica.

GAMBA, S. 2008. ¿Qué es la perspectiva de género y los estudios de género? "Diccionario de estudios de Género y Feminismos". Editorial Biblos.

OSORIO, R. s/d. Las mujeres investigadoras en educación; sus logros y retos.

RíOS, M. 2005. El impacto de los procesos de globalización en las representaciones científicas de las y los investigadores de la UNAM. In: GLAZMAN, R. \& FLORES, J. (eds.) Ciencia, Tecnología y Género en Iberoamericana. México: UNAM.

ZUBIETA, J., ROSAS, R. \& ABARCA, G. 2005. Aunque la mona tenga posgrados, mona se queda: la falta de equidad de género en el mercado laboral. In: BLAZQUEZ, N. \& FLORES, J. (eds.) Ciencia, tecnología y género en Iberoamérica. México: UNAM. 
